\title{
The Influence of Gravity on 3D Printing with the FFF/FDM Technique - Reverse FDM/FFF 3D Printing
}

\author{
Robert E. Przekop ${ }^{1, *}$, Daria Pakuła ${ }^{2}$, Miłosz Frydrych ${ }^{2}$, Marek Jałbrzykowski ${ }^{1,3}$ \\ Centre for Advanced Technologies, Adam Mickiewicz University in Poznan, 61-614 Poznan, Poland \\ Faculty of Chemistry, Adam Mickiewicz University in Poznan, 61-614 Poznan, Poland \\ Faculty of Mechanical Engineering, Bialystok University of Technology, Wiejska 45 C, $15-351$ Bialystok, Poland
}

Abstract: Often, simple questions do not have obvious answers and require an experiment that yields an unexpected answer. Have you ever wondered what will happen if you turn 180 degrees to the commonly accepted FDM printing convention? The paper presents the verification of the possibility of using the reverse orientation of the print head in traditionally used 3D printers of FFF/FDM systems. It has been shown that the mechanical parameters of the printed object can be even higher when reverse printing is used. The presented concept opens up new possibilities of designing printers in FFF/FDM systems, which use changed heat convection and the reverse effect of gravity on the printed object. This work was carried out using the TRIZ methodology.

Keywords: 3D printing, gravity, microgravity, gravitation influence, nozzle orientation, printing direct orientation, FDM, FFF, TRIZ.

\section{Introduction}

3D printing of thermoplastics has been known to science and technology for nearly 30 years [1-2]. The fundamental discovery of SLS [3] guided the further development of this technology. Subsequent solutions were based on the concept of incremental forming, layer by layer, and established the orientation of the processing tool [4].

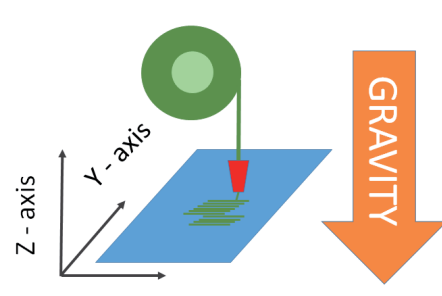

$\mathrm{X}$ - axis

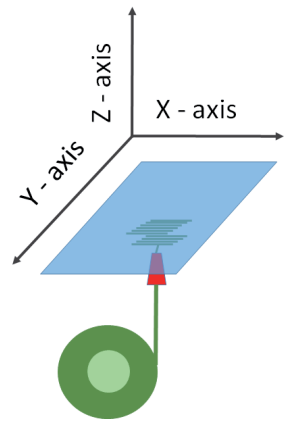

Figure 1. The concept of inversion of the extruder position convention in FFF/FDM printers.

A common convention in the design of thermoplastic printers in the FFF/FDM technique is that the extrusion micro-extruder is oriented horizontally in the direction of gravity. This convention results in part from the belief that the extruded material interacts with gravity which directs its growth. On the other hand, it seems to be completely consistent with human nature to orientate the work of the tool taking into account the direction of the gravitational field. This is how hydraulic presses 
work, as well as steam engines, in which the first piston systems were oriented, etc. However, innovations in science and technology were based on a completely different approach, which is why, for example, the Wankel engines or boxer engines were created. For fuse deposition modeling of thermoplastics using the micro-extrusion method, the approach that the machine table is below the micro-extruder has become established [5]. A critical analysis of the 3D printer design method using the TRIZ methodology [6] showed us that changing the concept of extruder orientation may bring new and useful knowledge. In this work, we want to show what will happen if we break the traditional principle (according to TRIZ) of designing FDM/FFF printers and reverse the orientation of the layer embossing head. It turns out that it is possible to design the construction of printing systems in the reverse way (3D printing reverse), which gives a lot of new possibilities from an engineering point of view. It also shows that in conditions of microgravity or gravity $=0$ the printer from this system can work with equal success. The tested material strength properties (PLA) show no differences depending on the gravitational orientation of the system, and what is more, there may be positive effects (slight increase in parameters) caused by an increase in the consistency of the obtained sample. The effects of heat convection, which run in a different orientation, are also important. This communication aims to inspire machine designers and 3D printing scientists.

\section{Experimental Section}

\subsection{Materials}

Polylactide (PLA) type Ingeo 2003D was purchased from NatureWorks (Minnetonka, MN, USA). Using a 3D printer FlashForge Finder two types of samples were printed by FDM: oars and bars, according to PN-EN- ISO 527-2. Parameters of printing are given in Table 1.

Table 1. Process parameters for sample printing.

\begin{tabular}{|l|l|l|l|}
\hline Layer height & $0.18 \mathrm{~mm}$ & Infill density & $100 \%$ \\
\hline Top layer height & $0.27 \mathrm{~mm}$ & Infill pattern & Grid \\
\hline Shells & 2 & Printing speed & $60 \mathrm{~mm} / \mathrm{s}$ \\
\hline $\begin{array}{l}\text { Top and bottom } \\
\text { layers number }\end{array}$ & 3 & Idle speed & $80 \mathrm{~mm} / \mathrm{s}$ \\
\hline $\begin{array}{l}\text { Bottom layers } \\
\text { number }\end{array}$ & 3 & Extruder temp. & $220^{\circ} \mathrm{C}$ \\
\hline
\end{tabular}

For flexural and tensile strength tests, the obtained materials were printed into type 1B dumbbell specimens in accordance with EN ISO 527:2012 and EN ISO 178:2006. Tests of the obtained specimens were performed on a universal testing machine INSTRON 5969 with a maximum load force of $50 \mathrm{kN}$. The traverse speed for tensile strength measurements was set at $2 \mathrm{~mm} / \mathrm{min}$, and for flexural strength was also set at $2 \mathrm{~mm} / \mathrm{min}$. Charpy impact test (with no notch) was performed on a InstronCeast 9050 impact-machine according to ISO 179-1. For all the series, 6 measurements were performed. Surface structure were analysed under Digital Light Microscope Keyence VHX 7000 with 100- $\times 1000 \mathrm{VH}-\mathrm{Z} 100 \mathrm{~T}$ lens. All of the pictures were recorded with a VHX 7020 camera.

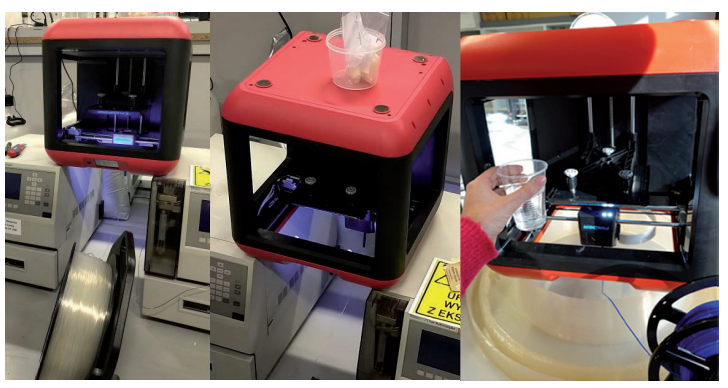

Figure 2. Printer FlashForge Finder reversed 3D printing.

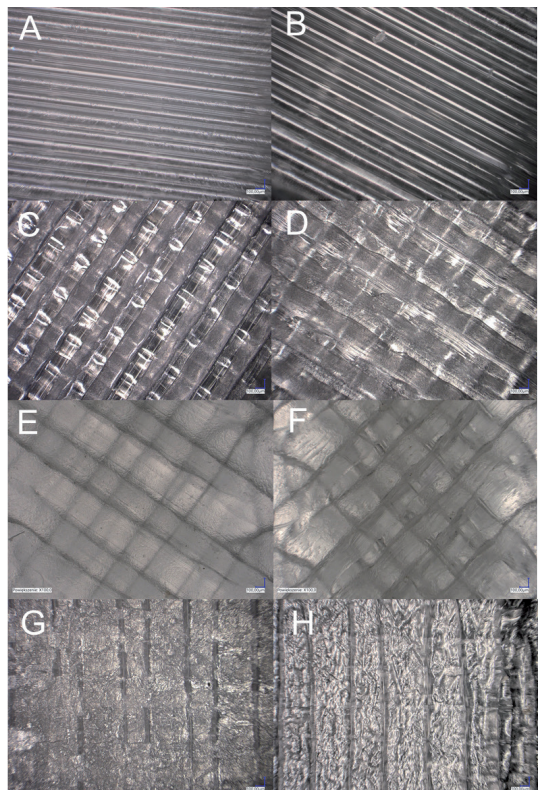

Figure 3. Optical microscopic images - normal gravity samples (NG) and reversed gravity samples (RG) A) RG sideway, B) NG sideway, C) RG top, D) NG top, E) RG bottom, F) NG bottom, G) RG bottom, H) NG bottom 


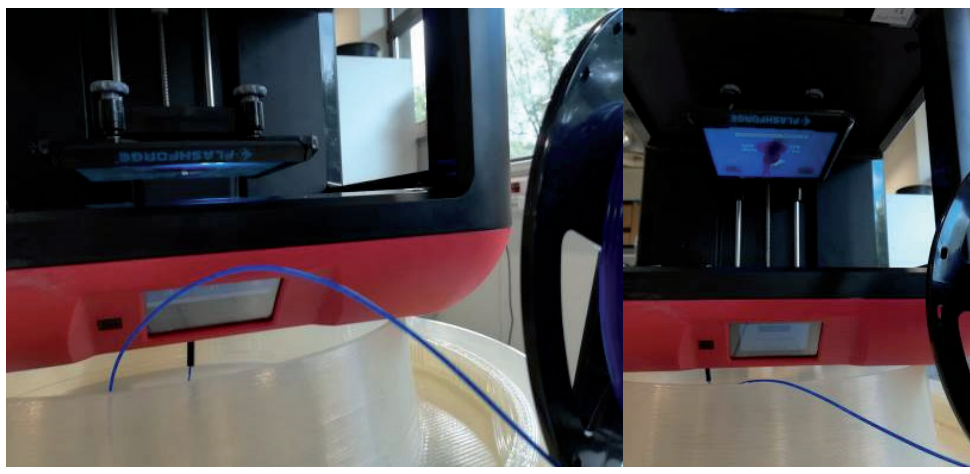

Figure 4. Printer FlashForge Finder reversed 3D printing.

\section{Results and Discussion}

Mechanical tests were carried out for the samples obtained by both traditional 3D printing and reverse 3D printing. For the samples, the tensile load was applied parallelly to the plane of layer-bylayer deposition. Table 2 summarizes the results of the tensile and bending strength tests as well as the impact tests. The measurements show that both the traditional method and the reversed method are characterized by similar mechanical properties.

Table 2. Mechanical properties of obtained sample

\begin{tabular}{|l|l|l|l|l|}
\hline & \multicolumn{2}{|l|}{ normal } & \multicolumn{2}{l|}{ reverse } \\
\hline & Value & $\begin{array}{l}\text { SD } \\
{[+/-]}\end{array}$ & Value & $\begin{array}{l}\text { SD } \\
{[+/-]}\end{array}$ \\
\hline Tensile strength [MPa] & 54.51 & 3.04 & 55.71 & 2.63 \\
\hline Elongation at break $[\mathrm{mm} / \mathrm{mm}]$ & 0.025 & 0.001 & 0.025 & 0.001 \\
\hline Flexural modulus [MPa] & 3180.6 & 109.9 & 3381.8 & 158.5 \\
\hline Flexural strength $[\mathrm{MPa}]$ & 86.34 & 4.28 & 88.62 & 6.01 \\
\hline Impact strength $\left[\mathrm{kJ} / \mathrm{m}^{2}\right]$ & 14.93 & 3.95 & 14.83 & 3.98 \\
\hline
\end{tabular}

Slightly higher values of tensile strength, flexural modulus, flexural strength are noticeable for samples obtained from reverse 3D printing. This may be due to better consistency in the structure of the tested material, and thus smaller defects in the samples. This is confirmed by Optical microscopic images (Figure 3) showing slight changes in the structure of the obtained materials. A noticeable difference can be observed between $C$ (reverse 3D printing) and D (traditional 3D printing) photos, which indicate a more clearly weave of reverse samples compared to the sample obtained using the traditional method. This is due to the fact that during reverse printing, the last layer of the sample is subjected to gravity and the hardened thermoplastic material does not flow over the printed object.

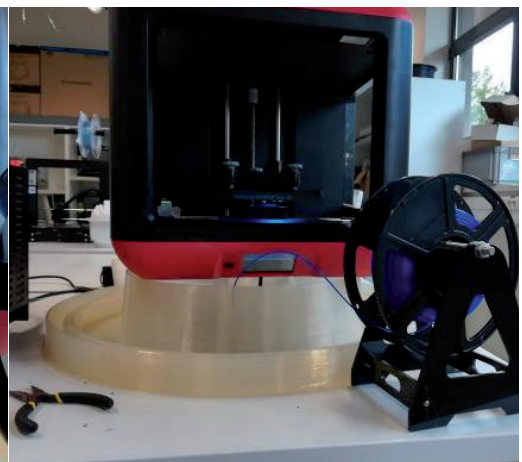

4. Conclusions

The paper presents a comparison between two printing methods: standard and reverse printing. Mechanical tests (tensile and bending strength, impact strength) were carried out in order to compare the mechanical properties of the obtained materials. The analyses indicate the potential possibilities of using reverse printing to obtain new materials. The materials obtained using two methods are characterized by similar values of mechanical strength. Slightly higher values can be seen for samples printed with the inverted method, which is due to smaller defects in the structure. The changes in the structure on the surface are presented in microscopic photos. The reverse printing method gives new possibilities in the design of 3D printers. By reversing the system, we obtain a change in heat convection in the case of a (heated table) and a different temperature distribution in the object in printers with a heated chamber. These are just some of the possibilities offered by the change in the orientation concept of the head and the printed object. Despite the small size of the printers' working fields, and thus the dimensions of printed objects, the influence of gravity on the processing process is significant and should be the subject of further research.

\section{Acknowledgments}

This work is supported by statutory funds from Centre for Advanced Technology Adam Mickiewicz University in Poznan.

\section{References and Notes}

1. Shahrubudin, N.; Lee, T.C.; Ramlan, R.; 2019. An Overview on 3D Printing Technology: Technological, Materials and Applications, Procedia Manufacturing, 35, 1286-1296.

2. Holzmann, P.; Breitenecker, R.J.; Soomro, A.A.; Schwarz, E.J.; 2017. User entrepreneur business models in 3D printing", 
Journal of Manufacturing Technology Management, 28, 1, 75-94.

3. Gibson, I.; Rosen, D.W.; Stucker, B.; 2010. Additive Manufacturing Technologies; Springer: Berlin/Heidelberg, Germany; 299-332.

4. Grimm, T.; 2002. Fused Deposition Modeling: A Technology Evaluation; T.A. Grimm \& Associates Inc.: Atlanta, GA, USA.

5. Tian, J.; Zhang, R.; Yang, J.; Chou, W.; Xue, P.; Ding, Y.; 2021. Additive Manufacturing of Wood Flour/PHAComposites Using Micro-ScrewExtrusion: Effect of Device and Process Parameters on Performance.; Polymers, 13, 1107

6. Ismail Ekmekcia, I.; Nebatia, E.E.; 2019. Triz Methodology and Applications Procedia Computer Science, 158, 303-315. 